www.richterzeitung.ch

Andrea Kaminski

\title{
15 Jahre «Justice - Justiz - Giustizia» - Pubertät durchlebt, Reife erworben
}

Beitragsart: Jubiläum

Zitiervorschlag: Andrea Kaminski, 15 Jahre «Justice - Justiz - Giustizia» - Pubertät durchlebt, Reife erworben, in: «Justice - Justiz - Giustizia» 2020/4 
[1] 15 Jahre Schweizer Richterzeitung «Justice - Justiz - Giustizia» ist ein Grund zum Feiern. Zumal wir als Herausgeber und Redakteure der deutschen Zeitschrift Betrifft JUSTIZ uns quasi als Patin oder Spiritus Rektor fühlen dürfen. Denn als Thomas Stadelmann und seine Mitstreiter die Schweizer Richterzeitung ins Leben riefen, haben sie ausdrücklich Betrifft JUSTIZ als Anregung genannt, auch für die Schweizer Richterinnen und Richter und alle an der Justiz Interessierten ein entsprechendes Blatt zur Verfügung zu stellen. Moderner als wir, die wir Betrifft JUSTIZ nach wie vor primär als Papier-Zeitschrift herausgeben. Inhaltsreich und nicht nur auf die Schweiz fixiert gibt es jede Menge spannende Artikel, die auch für die deutsche Klientel ausserordentlich interessant sind. Dafür sorgt die breit aufgestellte Redaktion mit Richterinnen und Richtern aller Stufen und aus allen Rechtsgebieten sowie Hochschullehrern.

[2] Dementsprechend übernehmen wir gelegentlich Artikel aus «Justice - Justiz - Giustizia» und freuen uns, dass wir immer wieder interessante Anregungen aufnehmen können und Autoren für unsere Zeitschrift finden, die wir ohne die Schweizer Kollegen nie gefunden hätten. Der Blick über die Grenzen und der Austausch mit Fragen «Wie macht ihr das? Wie geht ihr mit jenem Problem um oder mit jener (berufs-)ethischen Frage?» - das hilft uns allen, besser zu werden in unserem Beruf, und tut ganz einfach gut. Und so manche Thematik, auf die wir noch nicht gekommen sind, wird uns so vermittelt.

[3] Unsere Justiz-Systeme sind in vielem gleich, in vielem aber auch sehr verschieden. So können wir voneinander lernen, wie man ähnliche Fragestellungen auch anders lösen kann als wir es jeweils in unserem Land gewohnt sind.

[4] Zuletzt hat Corona die Schweizer Justiz ebenso wie die deutsche herausgefordert. Was den Einsatz von Videokonferenzen angeht, hat uns die Schweizer Richterzeitung durch ein Webinar vorgemacht, wie man damit umgehen könnte. Wir bei Betrifft JUSTIZ haben intensiv berichtet, welche (geringen) Erfahrungen es bereits in Deutschland gibt und haben dokumentiert, wie insbesondere angelsächsische Länder viel unbefangener und frischer die Möglichkeiten der digitalen Technik nutzen, um einen Stillstand der Justiz zu vermeiden, lange Anreisen für kurze Vernehmungen zu sparen und interessegerecht elektronisch schneller zu einem Ergebnis zu kommen, wenn der konkrete Fall geeignet ist.

[5] Also Fazit: die «Justice - Justiz - Giustizia» ist ein grosser Gewinn für uns alle, die wir die Justiz besser machen wollen. Wir von der Redaktion von Betrifft JUSTIZ gratulieren unserem erwachsenen Patenkind sehr sehr herzlich zum 15. Geburtstag und wünschen noch viele Jahre gute Ideen, viel Kraft für die Arbeit und begeisterte Leserinnen und Leser.

Andrea Kaminski, Direktorin des Amtsgerichts Velbert a.D. und Mitglied der Redaktion und des Herausgebervereins der Zeitschrift Betrifft JUSTIZ. 